\title{
Intensive Versus Conventional Insulin Therapy in Critically III Neurologic Patients: Still Searching for the Sweet Spot
}

\author{
Jennifer A. Frontera
}

Published online: 23 October 2010

(C) Springer Science+Business Media, LLC 2010

There are very few treatments in the world of critical care that have been shown to reduce mortality in well constructed randomized controlled trials. In fact, only therapeutic hypothermia after cardiac arrest, goal directed therapy for sepsis, low tidal volumes in ALI/ARDS, recombinant activated protein $\mathrm{C}$ in sepsis, and early tracheostomy in critically ill medical patients have substantial support in the literature [1-6]. Until recently, intensive glucose control was counted among this small group of therapeutic tools within the intensivist's arsenal.

In 2001, the landmark van den Berghe single-center SICU trial [7] generated intense interest when it revealed a reduction in ICU mortality from $8 \%$ in the conventional group to $4.6 \%$ in the intensive insulin group $(P=0.005)$, using intensive insulin infusion targeted to a blood glucose of 80-110 mg/dl compared to conventional insulin infusion initiated for a blood glucose $>215 \mathrm{mg} / \mathrm{dl}$ and targeted to 180-200 mg/dl. This study additionally demonstrated a $34 \%$ reduction in hospital mortality in the intensive insulin group $(P=0.01)$ as well as significant reductions in blood stream infections, acute renal failure, transfusion requirement, and critical illness polyneuropathy. The bulk of the mortality benefit in this study occurred in cardiothoracic patients who spent $>5$ days in the ICU.

The follow-up Leuven single-center trial of Medical ICU patients [8] was disappointing, as it demonstrated no difference in hospital mortality between the intensive insulin and conventional group. In fact, mortality among patients staying $<3$ days was actually higher in the

\section{J. A. Frontera $(\square)$}

Neuroscience Intensive Care Unit, Departments of Neurosurgery and Neurology, Mount Sinai School of Medicine, One Gustave Levy Place, Box 1136, New York, NY 10029, USA

e-mail: Jennifer.Frontera@mountsinai.org intensive insulin group (56 vs. $42 \%$ in the conventional group, $P=0.05$ ), though this effect lost when withdrawal of care patients were removed from analysis. In patients staying $>3$ days, mortality was $52.5 \%$ in the conventional group versus $43 \%$ in the intensive group $(P=0.009)$. Morbidity was reduced in the entire cohort including decreased acute renal failure, accelerated weaning from mechanical ventilation, and accelerated discharge from the ICU and hospital.

The NICE-SUGAR study [9] was the first completed multi-center mixed ICU population study to adequately address the issue of tight versus conventional glucose control and the effects of hypoglycemia. This study randomized 6,104 patients to intensive insulin therapy targeted to $91-108$ vs. $\leq 180 \mathrm{mg} / \mathrm{dl}$ and found increased mortality $(27.5 \%)$ in the intensive group compared to the conventional group $(24.9 \%, P=0.02)$ with a severe hypoglycemia rate $(\leq 40 \mathrm{mg} / \mathrm{dl})$ of $6.8 \%$ in the intensive group and $0.5 \%$ in the control arm $(P<0.001)$.

Interest in very tight glucose control waned after publication of this well designed study, but the question still remains: are critically ill neurological patients different from the general mixed ICU population? On the one hand, hyperglycemia is very common in critically ill neurological patients and has been shown to predict poor outcome. In general, hyperglycemia is associated with poor outcomes among various critically ill populations, but has the highest adjusted odds ratios for mortality among cardiac and neurological patients [10]. Elevated admission glucose and prolonged hyperglycemia have been linked to worse outcomes in patients with ischemic stroke, intracerebral hemorrhage, subarachnoid hemorrhage, and traumatic brain injury [11-18]. On the other hand, little is known regarding how well patients with acute brain injury tolerate hypoglycemia, a frequent side-effect of tight glucose 
control. Under normal conditions the ratio of brain to blood glucose is $0.6-0.7$, similar to CSF to serum glucose ratios. This ratio may be as low as $0.2-0.4$ in acute brain injury. Smaller reductions in serum glucose may lead to inadequate brain glucose supply in the context of acute brain injury [19]. Though microdialysis studies [20] in traumatic brain injury suggest that intensive insulin therapy compared to loose glucose control can lead to significant reductions in brain glucose with elevations in brain lactate/ pyruvate and glutamate (markers of cellular distress), there have been few randomized studies comparing intensive to conventional glucose control in a critically ill neurological population [19].

In this month's issue of Neurocritical Care, Green et al. publish the results of a small randomized trial of intensive insulin therapy (IIT) targeted to $80-110 \mathrm{mg} / \mathrm{dl}$ compared to conventional glucose control targeted to $<150 \mathrm{mg} / \mathrm{dl}$ in critically ill neurological patients [21]. This is one of the few randomized trials of glucose control to focus solely on critically ill neurological patients. Van den Berghe published a substudy of the SICU trial that included a small neurological cohort of 63 patients (roughly $4 \%$ of the 1,548 patients in the larger trial) [22]. In this study, there was no difference in 6 or 12 month outcome in the intensive compared to the conventional group, though the intensive therapy group made greater neurologic improvement between 6 and 12 months, had less critical illness polyneuropathy, fewer seizures and lower median ICP. GISKUK [23] was a study of 899 stroke patients randomized to glucose-potassium-insulin infusion with a goal glucose of $72-126 \mathrm{mg} / \mathrm{dl}$ versus saline infusion for $24 \mathrm{~h}$. This study, however, excluded critically ill neurological patients such as those with SAH, posterior circulation strokes, or coma. GIST-UK failed to demonstrate a difference in mortality or outcome at 90 days, but since the study was stopped for slow enrollment, it enrolled less than $50 \%$ of the projected 2,355 patients and was thus underpowered. Additionally, the mean glucose difference between the GIK and saline group was only $10 \mathrm{mg} / \mathrm{dl}$, which may not be clinically significant and the $24 \mathrm{~h}$ of treatment may have not been long enough to illicit an outcome effect.

The Green et al. study [21] enrolled mechanically ventilated, neurologically critically ill patients $(80 \%$ of whom had TBI, ICH, or SAH) expected to have an ICU length of stay of at least 3 days. Using these inclusion criteria, only $6 \%$ of screened patients were enrolled, which unfortunately leads to a rather small $N$ of 81 and an overall underpowered study (to detect a $40 \%$ reduction in mortality 540 patients would need to be enrolled). Nonetheless, some important observations were made in this trial.

First, there was a trend towards higher mortality in the IIT group (36 vs. $25 \%, P=0.34$ ) and no difference in functional outcome as measured by the modified Rankin scale, examining mRS $0-2$ vs. 3-6 or 0-3 vs. 4-6, even after excluding patients with poor baseline functional status. This is one of the first studies to, a priori, examine functional outcome, rather than simple mortality rates. The study population, overall, was very ill with a mean admission GCS of 6.8 in the IIT group and 6.9 in the conventional treatment group $(P=0.84)$. The outcomes at 90 days are similarly poor overall with severe disability or death occurring in $64 \%$ of patients in the IIT arm and 59\% of patients in the conventional arm $(P=0.81)$. Since withdrawal of care was a major contributor to death in several cases and an analysis excluding these patients was not performed, it is conceivable that with a less ill population the effects of IIT might be different.

Second, as seen in other trials, there was a five-fold increase in moderate hypoglycemia $(<60 \mathrm{mg} / \mathrm{dl}, P<$ 0.001 ), though no significant difference in severe hypoglycemia ( $<40 \mathrm{mg} / \mathrm{dl}$ ). In general, a glucose control study is only as good as the glucose control protocol. In the Green study, arterial blood glucose samples were primarily used, though capillary finger sticks, which have been shown to be imprecise in critical illness [24, 25], were allowed. Titration of the insulin infusion in the IIT group followed computer directed algorithm software provided by Glucommander (Glucotec Inc., Greenville, South Carolina). The conventional group received sliding scale subcutaneous insulin primarily, but could cross over to insulin infusion. Using this system, the mean average glucoses differed significantly between the two groups $(112 \mathrm{mg} / \mathrm{dl}$ in the IIT arm and $143 \mathrm{mg} / \mathrm{dl}$ in the conventional arm, $P<0.001$ ), however, the IIT group was more likely to have out of range glucose values. In fact, the glucose level was below range $8 \%$ of the time in the IIT group (compared to $1.3 \%$ in the conventional group) and above range $46 \%$ of the time (compared to $35 \%$ in the conventional group). While the mean average glucoses may differ significantly between groups, this may be due to outlying glucose measurements. Of greater interest would be the glucose burden over time, or the area under the curve, to assess how much time each group spent within a certain glucose range. Since the conventional group received blood glucose checks every $6 \mathrm{~h}$ (compared to every $1-2 \mathrm{~h}$ in the IIT group), it would be difficult to reconstruct a comparative glucose burden analysis. As with the GIST-UK study, the mean average glucose in the two groups differed by a small amount: only $31 \mathrm{mg} / \mathrm{dl}$. This may represent a clinically insignificant glucose difference. Another critical factor in analyzing glucose control studies is to assess the feeding protocols in each arm. Since $87 \%$ of patients in this study received enteral feeds within 1 day of study enrollment, and none of the hypoglycemic episodes recorded occurred while a patient was NPO, the authors successfully eliminated this confounder from their study. 
Also in this month's issue of Neurocritical Care, Graffagnino et al. publish the results of a retrospective review of an intensive insulin therapy protocol targeted to a blood glucose of $80-120 \mathrm{mg} / \mathrm{dl}$ compared to historical controls targeted to $<150 \mathrm{mg} / \mathrm{dl}$ in a critically ill population treated in neuro-ICU setting [26]. Though demographically the tight and loose insulin control groups appear similar, only a quarter of the patients had an admitting diagnosis of $\mathrm{ICH}$, $\mathrm{SAH}, \mathrm{TBI}$, or ischemic stroke in each group. The remaining $\sim 75 \%$ of patients had primary admitting diagnoses of "Other" and it remains unclear what population was primarily studied in this paper. Glucose control in either group could be achieved with insulin infusion or adjusted multidosing. Though the two groups had significantly differed median glucose values (126 vs. $137 \mathrm{mg} / \mathrm{dl})$, this difference is clinically insignificant and there was no difference in the mean of means, nor the mean of medians. What is interesting about this paper is the increased hypoglycemia $(4.6 \%$ of tight control group and $2.3 \%$ in loose control group $<40 \mathrm{mg} / \mathrm{dl}$ ) and increased mortality (11\% in tight control group vs. $7.9 \%$ in loose control group; $P=0.0013$ ) observed during the epoch when tight glucose control was targeted. Indeed, irrespective of insulin protocol, the odds of dying increased incrementally with more severe hypoglycemia on univariate analysis. This effect remained after controlling for admitting diagnoses, which varied significantly between the time epochs. Since admission metrics of neurological and critical illness (i.e., GCS, APACHE scores, etc.) were not accounted for, it is impossible to assign a causal relationship between hypoglycemia and increased mortality without a multivariate analysis. Interestingly, the authors found that hyperglycemia was more common in the tight control group and was also associated with increased mortality. It is conceivable that the increased mortality during the tight control epoch was due, in part, to hyperglycemia or even fluctuations in glucose levels. Perhaps the most important feature of this study is that it provides a real life example of the potential pitfalls of instituting a tight glucose control protocol. To wit: the best intentioned glucose control algorithms can lead to increased hypoglycemia without a clinically significant difference in hyperglycemia rates.

The balancing act of mitigating the negative effects of hyperglycemia, while not incurring additional injury due to hypoglycemia, has been a challenge encountered in several other studies. The most concerning criticism of the Leuven trials [7, 8] has been the shocking rate of hypoglycemia $<40 \mathrm{mg} / \mathrm{dl}$, which occurred in $18.7 \%$ of intensive insulin treated patients $(25 \%$ of those staying $>3$ days in the ICU) and $3.1 \%$ of conventionally treated patients in the MICU study. Unacceptable rates of hypoglycemia led to the early termination of both the VISEP [27] and Glucontrol [28] studies. The VISEP study [27] found severe hypoglycemia in $17 \%$ in the intensive insulin group versus $4 \%$ in the conventional group. Despite such rates of hypoglycemia, no difference in mortality or organ failure rates were found at 28 days. Similarly, the Glucontrol study [28], which found an $8.7 \%$ rate of hypoglycemia $<60 \mathrm{mg} / \mathrm{dl}$ in the intensive insulin group compared to $2.7 \%$ in the conventional group $(P<0.0001)$, found no difference in mortality. Since both VISEP and Glucontrol were prematurely terminated, it is not surprising that both were underpowered to detect a difference between intensive versus conventional glucose control. Additionally, as none of these studies examined cognitive outcomes, it is difficult to know the real impact of iatrogenic hypoglycemia on neurological outcome. Indeed, none of the studies even differentiate iatrogenic hypoglycemia from symptomatic hypoglycemia due to sepsis, renal, or liver failure, which may auger a worse outcome.

Overall, though the Green et al. study [21] was well designed and executed, we still await a definitive fully powered study of critically ill neurological patients. Aside from mortality and modified Rankin outcomes, a study which also examines cognitive outcome would add a great deal to our understanding of the effects of both hyper- and hypoglycemia. While very high glucose levels seem imprudent, and levels of hypoglycemia that lead to short term neurologic compromise are also inadvisable, the sweet spot for targeted glucose control in critically ill neurological patients remains elusive. For now, the AHA guidelines, which suggest initiation of glycemic control for glucose values $>140 \mathrm{mg} / \mathrm{dl}$ (Class IIa, Level C), seem reasonable [29]. The best method of achieving this goal (i.e., sliding scale insulin or insulin infusion) remains unclear, but must take into account cost, labor and patient comfort. In the future, closed loop methods of continuous blood glucose measurement followed by insulin delivery may allow for effective control of hyperglycemia with minimal risk of hypoglycemia. Only when optimal methods of controlling glucose without inducing hypoglycemia are available, can we proceed with the necessary large randomized controlled trials to assess the effects of adequate glucose control on outcome.

Disclosures The authors have nothing to disclose.

\section{References}

1. The Acute Respiratory Distress Syndrome Network. Ventilation with lower tidal volumes as compared with traditional tidal volumes for acute lung injury and the acute respiratory distress syndrome. N Engl J Med. 2000;342:1301-8.

2. The Hypothermia after Cardiac Arrest Study Group. Mild therapeutic hypothermia to improve the neurologic outcome after cardiac arrest. N Engl J Med. 2002;346:549-56. 
3. Bernard GR, Vincent JL, Laterre PF, et al. Efficacy and safety of recombinant human activated protein $\mathrm{C}$ for severe sepsis. $\mathrm{N}$ Engl J Med. 2001;344:699-709.

4. Bernard SA, Gray TW, Buist MD, et al. Treatment of comatose survivors of out-of-hospital cardiac arrest with induced hypothermia. N Engl J Med. 2002;346:557-63.

5. Rivers E, Nguyen B, Havstad S, et al. Early goal-directed therapy in the treatment of severe sepsis and septic shock. N Engl J Med. 2001;345:1368-77.

6. Rumbak MJ, Newton M, Truncale T, Schwartz SW, Adams JW, Hazard PB. A prospective, randomized, study comparing early percutaneous dilational tracheotomy to prolonged translaryngeal intubation (delayed tracheotomy) in critically ill medical patients. Crit Care Med. 2004;32:1689-94.

7. Van den Berghe G, Wouters P, Weekers F, et al. Intensive insulin therapy in the critically ill patients. N Engl J Med. 2001;345: 1359-67.

8. Van den Berghe G, Wilmer A, Hermans G, et al. Intensive insulin therapy in the medical ICU. N Engl J Med. 2006;354:449-61.

9. Finfer S, Chittock DR, Su SY, et al. Intensive versus conventional glucose control in critically ill patients. N Engl J Med. 2009;360: 1283-97.

10. Whitcomb BW, Pradhan EK, Pittas AG, Roghmann MC, Perencevich EN. Impact of admission hyperglycemia on hospital mortality in various intensive care unit populations. Crit Care Med. 2005;33:2772-7.

11. Baird TA, Parsons MW, Phanh T, et al. Persistent poststroke hyperglycemia is independently associated with infarct expansion and worse clinical outcome. Stroke. 2003;34:2208-14.

12. Bruno A, Levine SR, Frankel MR, et al. Admission glucose level and clinical outcomes in the NINDS rt-PA Stroke Trial. Neurology. 2002;59:669-74.

13. Demchuk AM, Morgenstern LB, Krieger DW, et al. Serum glucose level and diabetes predict tissue plasminogen activatorrelated intracerebral hemorrhage in acute ischemic stroke. Stroke. 1999;30:34-9.

14. Frontera JA, Fernandez A, Claassen J, et al. Hyperglycemia after SAH: predictors, associated complications, and impact on outcome. Stroke. 2006;37:199-203.

15. Jeremitsky E, Omert LA, Dunham CM, Wilberger J, Rodriguez A. The impact of hyperglycemia on patients with severe brain injury. J Trauma. 2005;58:47-50.

16. Kase CS, Furlan AJ, Wechsler LR, et al. Cerebral hemorrhage after intra-arterial thrombolysis for ischemic stroke: the PROACT II trial. Neurology. 2001;57:1603-10.

17. Kimura K, Iguchi Y, Inoue T, et al. Hyperglycemia independently increases the risk of early death in acute spontaneous intracerebral hemorrhage. J Neurol Sci. 2007;255:90-4.
18. Wartenberg KE, Schmidt JM, Claassen J, et al. Impact of medical complications on outcome after subarachnoid hemorrhage. Crit Care Med. 2006;34:617-23; quiz 624.

19. Godoy DA, Di Napoli M, Rabinstein AA. Treating hyperglycemia in neurocritical patients: benefits and perils. Neurocrit Care. doi:10.1007/s12028-010-9404-8.

20. Vespa P, Boonyaputthikul R, McArthur DL, et al. Intensive insulin therapy reduces microdialysis glucose values without altering glucose utilization or improving the lactate/pyruvate ratio after traumatic brain injury. Crit Care Med. 2006;34:850-6.

21. Green DM, O'Phelan KH, Bassin SL, Chang CW, Stern TS, Asai SM. Intensive versus conventional insulin therapy in critically Ill neurologic patients. Neurocrit Care. doi:10.1007/s12028-010-9417-3.

22. Van den Berghe G, Schoonheydt K, Becx P, Bruyninckx F, Wouters PJ. Insulin therapy protects the central and peripheral nervous system of intensive care patients. Neurology. 2005;64: 1348-53.

23. Gray CS, Hildreth AJ, Sandercock PA, et al. Glucose-potassiuminsulin infusions in the management of post-stroke hyperglycaemia: the UK Glucose Insulin in Stroke Trial (GIST-UK). Lancet Neurol. 2007;6:397-406.

24. Critchell CD, Savarese V, Callahan A, Aboud C, Jabbour S, Marik P. Accuracy of bedside capillary blood glucose measurements in critically ill patients. Intensive Care Med. 2007;33: 2079-84.

25. Kanji S, Buffie J, Hutton B, et al. Reliability of point-of-care testing for glucose measurement in critically ill adults. Crit Care Med. 2005;33:2778-85.

26. Graffagnino C, Gurran AR, Kolls BJ, Olson DM. Intensive Insulin Therapy in the Neurocritical Care Setting is Associated with Poor Clinical Outcomes. Neurocrit Care. 2010;14, in press.

27. Brunkhorst FM, Engel C, Bloos F, et al. Intensive insulin therapy and pentastarch resuscitation in severe sepsis. N Engl J Med. 2008;358:125-39.

28. Preiser JC, Devos P, Ruiz-Santana S, et al. A prospective randomised multi-centre controlled trial on tight glucose control by intensive insulin therapy in adult intensive care units: the Glucontrol study. Intensive Care Med. 2009;35:1738-48.

29. Adams HP Jr, del Zoppo G, Alberts MJ, et al. Guidelines for the early management of adults with ischemic stroke: a guideline from the American Heart Association/American Stroke Association Stroke Council, Clinical Cardiology Council, Cardiovascular Radiology and Intervention Council, and the Atherosclerotic Peripheral Vascular Disease and Quality of Care Outcomes in Research Interdisciplinary Working Groups: The American Academy of Neurology affirms the value of this guideline as an educational tool for neurologists. Circulation. 2007;115: e478-534. 